\title{
Combinaciones de retroalimentación e igualación de la muestra generalizada bajo estímulos y relaciones de igualación familiares y no familiares
}

\author{
MARIO SERRANO* \\ Universidad Veracruzana, CEICAH, Veracruz, México \\ ORCID: https://orcid.org/0000-0002-1190-3024 \\ CARLOS FLORES \\ Universidad de Guadalajara, CEIC, Jalisco, México \\ ORCID: https://orcid.org/0000-0002-3085-4029
}

\begin{abstract}
How to quote this article: Serrano, M. \& Flores, C. (2019). Feedback combinations and generalized matching-to-sample performance under familiar and unfamiliar stimuli and matching relations. Acta Colombiana de Psicología, 22(1), 52-60. doi: http://www.dx.doi.org/10.14718/ACP.2019.22.1.4
\end{abstract}

Resumen

\begin{abstract}
Seis grupos de estudiantes de preparatoria se expusieron a una tarea de igualación de la muestra de segundo orden y a pruebas de generalización con estímulos familiares y no familiares, así como con una nueva relación de igualación. Para dos grupos, las respuestas de igualación correctas e incorrectas produjeron la retroalimentación correspondiente de acuerdo con un programa continuo y uno intermitente, respectivamente. Las respuestas correctas produjeron retroalimentación y las respuestas incorrectas produjeron pantallas en blanco y viceversa para otros dos grupos, respectivamente. Dos grupos adicionales estuvieron expuestos a combinaciones similares de retroalimentación y pantallas en blanco, pero se instruyó a los participantes sobre el "significado" de las pantallas antes del entrenamiento. Se observó igualación de la muestra generalizada extra-relacional con estímulos familiares o no familiares solo después de las condiciones de entrenamiento en las que se programó retroalimentación intermitente Correcto-Incorrecto, así como cuando las respuestas de igualación incorrectas produjeron pantallas en blanco y las respuestas correctas produjeron la retroalimentación correspondiente. Las instrucciones sobre el significado de las pantallas en blanco produjeron ejecuciones generalizadas ligeramente superiores a las observadas después de la retroalimentación continua Correcto-Incorrecto, las cuales a su vez fueron similares a las ejecuciones observadas después de la condición Correcto-Pantalla en blanco, sin instrucción. Los resultados confirman una tendencia inicial a tratar las pantallas en blanco como retroalimentación para respuestas correctas y sugieren un proceso común de "desligamiento" entre la retroalimentación intermitente y la retroalimentación Incorrecto-Pantalla en blanco.

Palabras clave: control abstracto del estímulo, discriminación condicional, igualación de la muestra generalizada, humanos.
\end{abstract}

\section{Feedback combinations and generalized matching-to-sample performance under familiar and unfamiliar stimuli and matching relations}

\begin{abstract}
Six groups of high-school students were exposed to a second-order matching-to-sample task and generalization tests trials using familiar and unfamiliar stimuli as well as a new matching relation. For two groups correct and incorrect matching responses produced the corresponding feedback according to continuous and intermittent schedules, respectively. Correct responses produced feedback and incorrect responses produced blanks and vice versa for other two groups, respectively. Two additional groups were exposed to similar feedback-blanks combinations but participants were instructed about the "meaning" of blanks before training. Extra-relational generalized matching-to-sample performance with either familiar or unfamiliar stimuli was observed after training conditions in which intermittent right-wrong feedback was scheduled, as well as when incorrect matching responses produced blanks and correct responses produced the corresponding feedback.
\end{abstract}

\footnotetext{
Universidad Veracruzana, Centro de Estudios e Investigaciones en Conocimiento y Aprendizaje Humano. Av. Orizaba N. ${ }^{\circ} 203$, Fraccionamiento Veracruz, Xalapa, Veracruz, México. C. P. 91020. Teléfono: +52 (228) 89034 65. mserrano@uv.mx

Partes de este trabajo se presentaron en la "42nd Annual Convention of the Association for Behavior Analysis International". La investigación se condujo durante el año sabático del primer autor en la Universidad de Guadalajara, bajo la supervisión del segundo autor. Se agradece al Dr. Oscar García Leal por facilitar la infraestructura para las actividades académicas y de investigación.
} 
Instructions about the meaning of blanks produced generalized performances slightly higher to those observed after continuous right-wrong feedback, which in turn were similar to performances observed after the uninstructed right-blank feedback combination condition. Results confirm an initial tendency to treat blanks as if they mean right and suggest a common "detachment" processes between intermittent feedback and the wrong-blanks feedback combination.

Keywords: abstract stimulus control, conditional discrimination, generalized matching-to-sample, humans.

\title{
Combinaçóes de retroalimentação e igualação da amostra generalizada sob estímulos e relaçóes de igualaçáo familiares e náo familiares
}

\author{
Resumo
}

\begin{abstract}
Seis grupos de estudantes de vestibular foram expostos a uma tarefa de igualação da amostra de segunda ordem e a testes de generalização com estímulos familiares e não familiares, bem como a uma nova relação de igualação. Para dois grupos, as respostas de igualação corretas e incorretas produziram a retroalimentação correspondente de acordo com um programa contínuo e um intermitente, respectivamente, em que as respostas corretas produziram retroalimentação e as incorretas, ecrãs brancos - e vice-versa para outros dois grupos. Dois grupos adicionais estiveram expostos a combinações semelhantes de retroalimentação e ecrãs brancos, mas foi instruído aos participantes o "significado" das telas antes do treinamento. Foi observada igualação da amostra generalizada extrarrelacional com estímulos familiares e não familiares somente depois das condições de treinamento nas quais foi programada a retroalimentação intermitente Correto-incorreto, bem como quando as respostas de igualação incorretas produziram ecrãs brancos, e quando as respostas corretas produziram a retroalimentação correspondente. As instruções sobre o significado dos ecrãs brancos produziram execuções generalizadas levemente superiores às observadas após a retroalimentação contínua Correto-incorreto, as quais, por sua vez, foram similares às execuções observadas depois da condição Correto-ecrã branco, sem instrução. Os resultados confirmam uma tendência inicial a tratar os ecrãs brancos como retroalimentação para respostas corretas e sugerem um processo comum de "desligamento" entre a retroalimentação intermitente e a retroalimentação Incorreto-ecrã branco.

Palavras-chave: controle abstrato do estímulo, discriminação condicional, igualação da amostra generalizada, humanos.
\end{abstract}

\section{Introducción}

En una situación de discriminación condicional, las funciones discriminativa y delta de los estímulos varían de un momento a otro dependiendo de un tercer segmento de estímulo anterior o concurrente. En términos del procedimiento típico de igualación de la muestra con palomas, descrito originalmente por Skinner (1950), el tercer segmento de estímulo es el estímulo de muestra, mientras que los estímulos de comparación son los objetos de estímulo cuyas funciones varían ensayo a ensayo. Sobre la base de las propiedades físicas de los estímulos (como identidad, diferencia, semejanza, etc.) se pueden entrenar diferentes relaciones de igualación, y en el caso de los seres humanos, después de aprender a desempeñarse de acuerdo con una o más relaciones de igualación, los participantes pueden igualar inmediatamente estímulos de muestra y comparación no familiares en concordancia con tales criterios. De ser el caso, se dice que la ejecución es generalizada debido a que no se restringe a los estímulos específicos entrenados ni sus propiedades físicas.
Al igual que en los escenarios naturales, responder bajo procedimientos de igualación de la muestra puede ser influido por la retroalimentación, entendida como estímulos exteroceptivos respecto de la correspondencia entre el comportamiento de un individuo y las contingencias de reforzamiento vigentes o pasadas (Mangiapanello \& Hemmes, 2015). Por ejemplo, en un estudio reciente, Hirst, DiGennaro-Reed y Reed (2013) exploraron los efectos de la precisión de la retroalimentación sobre la adquisición de una discriminación condicional arbitraria en adultos jóvenes. En su estudio, los grupos de participantes recibieron retroalimentación de que sus respuestas de igualación eran correctas después de elecciones incorrectas, y viceversa, en $25,50,75$ y $100 \%$ de los ensayos, y observaron que, a mayor proporción de ensayos con retroalimentación imprecisa, menor adquisición de una discriminación condicional en una segunda fase con retroalimentación precisa; lo que sugiere que la precisión de la retroalimentación establece una relación discriminativa entre la situación de estímulo y el comportamiento de manera similar a las instrucciones o los estímulos que especifican contingencias. En este sentido, Catania (2006) había señalado previamente 
que proporcionar información a un individuo sobre si su respuesta fue correcta es similar a decirle "responde de la misma manera la próxima vez", sugiriendo que "puede ser engañoso hablar de las propiedades reforzantes de estar en lo correcto y las propiedades de castigo de estar en lo incorrecto" (p. 298).

Utilizando una tarea de aprendizaje discriminativo verbal, Spence (1964) reportó que la ejecución a lo largo de los ensayos de entrenamiento fue menor bajo una combinación de retroalimentación Correcto-Nada que bajo una combinación de retroalimentación Incorrecto-Nada, que a su vez produjo ejecuciones similares a aquellas que se observaron bajo una condición en la que se utilizó la típica combinación de retroalimentación Correcto-Incorrecto. En línea con la idea expresada por Catania (2006), Spence (1964) sugirió que los efectos disímiles de las combinaciones de retroalimentación Correcto-Nada e Incorrecto-Nada se debían a las características "informativas" de $\mathrm{Nada}$ (i.e., pantallas en blanco) más que a una diferencia en las funciones de reforzamiento positivo y negativo de Correcto e Incorrecto, respectivamente. Más específicamente, Spence (1964) propuso que, en la ausencia de instrucciones sobre su significado, los participantes tienden a tratar las pantallas en blanco como si significaran Correcto bajo ambos tipos de combinaciones de retroalimentación. De ser de esa manera, dicha tendencia es incompatible con las demandas de la tarea experimental de tratar a las pantallas en blanco como Incorrecto bajo la combinación de retroalimentación Correcto-Nada, mientras que en la combinación de retroalimentación Incorrecto-Nada la tendencia promueve condiciones similares a aquellas en las que las respuestas correctas e incorrectas producen la retroalimentación correspondiente en cada ensayo.

Sin embargo, experimentos previos sobre igualación de la muestra generalizada sugieren que la ausencia de retroalimentación en algunos ensayos tiene un efecto más complejo sobre el comportamiento. Por ejemplo, Martínez y Ribes (1996) informaron que los participantes expuestos a una condición de realimentación intermitente Correcto-Incorrecto (cada tres ensayos), estuvieron menos controlados por instrucciones iniciales falsas con respecto a la relación de igualación correcta -tanto en el entrenamiento como en las pruebas de generalización-en comparación con participantes expuestos a retroalimentación continua Correcto-Incorrecto; una tercera condición de retroalimentación demorada (i.e., al final de cada sesión de entrenamiento) produjo ejecuciones intermedias. En este sentido, Martínez y Ribes (1996) sugirieron que la ausencia de retroalimentación en algunos ensayos de igualación promovía un "desligamiento" de las contingencias momento a momento y, en consecuencia, la autoconstrucción de un estímulo discriminativo verbal abstracto controlado por la propiedad funcional compartida por los estímulos a lo largo de los ensayos (i.e., la relación de igualación).

Utilizando tareas de igualación de la muestra con combinaciones de retroalimentación Correcto-Nada e Incorrecto-Nada, así como la típica condición de retroalimentación continua Correcto-Incorrecto, Serrano, García y López (2009) reportaron que la ejecución de igualación de la muestra generalizada concuerda con la hipótesis expresada por Spence (1964) de que los participantes tienden a tratar Nada como si significara Correcto a lo largo de los ensayos de entrenamiento. Y más recientemente, Serrano, Flores, Peralta y Martínez (2017) observaron una ejecución de igualación de la muestra generalizada más alta después de una condición de entrenamiento con una combinación de retroalimentación Incorrecto-Nada que después de una Correcto-Nada, así como que la combinación de retroalimentación Incorrecto-Nada derivó en una ejecución generalizada más alta que la retroalimentación continua Correcto-Incorrecto cuando las pruebas de generalización incluyeron una relación de igualación no entrenada. En virtud de la ejecución observada en tales ensayos de prueba de generalización "extra-relacionales", estos últimos autores sugirieron que sus resultados se debieron a la tendencia inicial de tratar Nada como si significara Correcto, pero también a un desligamiento respecto de las contingencias momento a momento similar al descrito por Martínez y Ribes (1996) respecto de la retroalimentación intermitente Correcto-Incorrecto. El experimento, sin embargo, no incluyó una condición en la que las respuestas correctas e incorrectas produjeran retroalimentación intermitente.

Ahora bien, el experimento del presente estudio se realizó para comparar los efectos de la combinación de retroalimentación Incorrecto-Nada versus la retroalimentación intermitente Correcto-Incorrecto sobre la ejecución de igualación de la muestra generalizada. Si los efectos de la combinación de retroalimentación Incorrecto-Nada son el resultado de una tendencia inicial a tratar Nada como si significara Correcto, pero igualmente a la elaboración de estímulos verbales debido a un desligamiento respecto de las contingencias momento a momento, los participantes entrenados bajo una combinación de retroalimentación Incorrecto-Nada deberían mostrar ejecuciones similares a las observadas para los participantes expuestos a la retroalimentación intermitente Correcto-Incorrecto, las que a su vez deberían ser mayores que las observadas para participantes entrenados bajo la retroalimentación continua Correcto-Incorrecto.

Debe destacarse que, en contraste con la hipótesis expresada por Spence (1964), ninguno de los experimentos disponibles sobre combinaciones de retroalimentación e igualación de la muestra generalizada incluyó participantes 
instruidos sobre el significado de las pantallas en blanco antes del entrenamiento; de modo que, siguiendo la sugerencia expresada por Catania (2006), si Correcto e Incorrecto tienen una función instruccional del tipo "responde (o no respondas) de la misma manera la próxima vez", los participantes instruidos sobre el significado de las pantallas en blanco y entrenados bajo las combinaciones de retroalimentación Correcto-Nada e Incorrecto-Nada deberán mostrar ejecuciones generalizadas similares a las observadas para los participantes expuestos a la retroalimentación continua Correcto-Incorrecto.

\section{Método}

\section{Participantes}

Se contó con la participación de 30 estudiantes de bachillerato de entre 17 y 18 años de edad, 21 mujeres y 9 hombres, experimentalmente ingenuos en tareas de igualación de la muestra, así como en cualquier otro procedimiento experimental en psicología. Todos los participantes dieron su consentimiento escrito para colaborar en el estudio. Aleatoriamente se conformaron seis grupos de cinco participantes cada uno para la realización del estudio: (a) Retroalimentación Continua Correcto-Incorrecto, (b) Retroalimentación Intermitente Correcto-Incorrecto, (c) Retroalimentación Correcto-Nada, (d) Retroalimentación Incorrecto-Nada, (e) Retroalimentación Correcto-Nada con Instrucciones y (f) Retroalimentación Incorrecto-Nada con Instrucciones.
Aparatos y situación experimental

Los participantes trabajaron individualmente en salones de $3 \mathrm{~m}^{2}$ aproximadamente, aislados de ruido y distractores. Cada uno de los cinco salones estuvo equipado con una mesa, una silla, una computadora de escritorio (modelo HP Compaq, dc5850) y un mouse. Los estímulos se presentaron a través del monitor de la computadora, y el mouse sirvió como dispositivo de respuesta. Los eventos experimentales se controlaron y registraron automáticamente con el software Superlab® 4.5 .

Para todos los grupos de participantes se utilizaron ensayos de igualación de la muestra de segundo orden simultáneos a lo largo del experimento, y en cada ensayo se desplegaron siete figuras coloreadas: dos figuras fungieron como estímulos de segundo orden, los cuales modelaron visualmente la relación de igualación en cada ensayo; una figura sirvió como estímulo de muestra; y las cuatro figuras restantes fungieron como estímulos de comparación.

Los estímulos de segundo orden se ubicaron en la parte superior del monitor de la computadora, mientras que los estímulos de muestra se ubicaron en el centro y los de comparación en la parte inferior, y siempre fueron diferentes en forma y color respecto de los estímulos de muestra y de comparación. En cada ensayo de igualación, un estímulo de comparación fue idéntico al estímulo de muestra, uno fue semejante en forma, otro fue semejante en color, y el cuarto fue diferente tanto en forma como en color. La posición espacial de cada tipo de estímulo de comparación cambió aleatoriamente de un ensayo a otro a lo largo del experimento.

Tabla 1.

Formas y colores utilizados para elaborar los estímulos de segundo orden y los arreglos de igualación de la muestra en cada fase del experimento

\begin{tabular}{|c|c|c|c|c|}
\hline \multirow{2}{*}{ Estímulos } & \multicolumn{2}{|c|}{ Ensayos de preprueba y generalización } & \multicolumn{2}{|c|}{ Entrenamiento } \\
\hline & Forma & Color & Forma & Color \\
\hline \multirow{6}{*}{$\begin{array}{c}\text { Segundo } \\
\text { Orden }\end{array}$} & Rombo & Azul Cielo & Rombo & Azul Cielo \\
\hline & Cruz & Dorado & Cruz & Dorado \\
\hline & Circulo & Negro & Circulo & Negro \\
\hline & Igual A & Gris & & \\
\hline & Chevron & Púrpura & & \\
\hline & Trapezoide & Rosa & & \\
\hline \multirow{6}{*}{$\begin{array}{l}\text { Estímulos de } \\
\text { muestra y } \\
\text { comparación }\end{array}$} & Triángulo & Verde & Triángulo & Verde \\
\hline & Cuadrado & Amarillo & Cuadrado & Amarillo \\
\hline & Pentágono & Rojo & Pentágono & Rojo \\
\hline & $\mathrm{L}$ & Azul & & \\
\hline & Paralelogramo & Anaranjado & & \\
\hline & Hexágono & Café & & \\
\hline
\end{tabular}


Tanto la preprueba como las pruebas de generalización constaron de 36 ensayos (12 de semejanza en color, 12 de semejanza en forma y 12 de diferencia), mientras que el entrenamiento constó de 72 ensayos ( 36 de semejanza en color y 36 de semejanza en forma). La Tabla 1 muestra las figuras y colores utilizados para generar los estímulos de segundo orden y los arreglos de igualación de la muestra de primer orden (i.e., un estímulo de muestra y cuatro estímulos de comparación) utilizados en cada fase del experimento.

Específicamente, un conjunto de tres figuras (rombo, cruz y círculo) y tres colores (azul claro, dorado y negro) permitieron la construcción de 18 parejas de estímulos semejantes en forma, 18 semejantes en color y 36 diferentes en forma y color; y otro conjunto de tres figuras (triángulo, cuadrado y pentágono) y tres colores (verde, amarillo y rojo) permitieron la construcción de 36 arreglos de igualación de la muestra de primer orden, de los cuales cada uno se presentó dos veces entre dos bloques de ensayos de entrenamiento. Las parejas de estímulos de segundo orden semejantes en color y las semejantes en forma se correlacionaron aleatoriamente con los arreglos de igualación de la muestra de primer orden en el primer bloque de ensayos de entrenamiento, mientras que las correlaciones entre los estímulos de segundo orden y los arreglos de igualación de la muestra se invirtieron entre los ensayos de entrenamiento del segundo bloque. Se extrajeron aleatoriamente seis parejas de estímulos diferentes en forma y color del tercer grupo de estímulos de segundo orden y se correlacionaron con seis arreglos de igualación de la muestra de primer orden, también extraídos aleatoriamente. Estos seis ensayos de igualación se utilizaron como parte de los ensayos de la preprueba y de la prueba de generalización.

Adicionalmente, un tercer conjunto de tres figuras (igual a, chevron y trapezoide) y tres colores (gris, morado y rosa) permitió la construcción de tres nuevos conjuntos de estímulos de segundo orden similares a los que se describieron anteriormente, y se construyó un segundo conjunto de 36 arreglos de igualación de la muestra de primer orden utilizando tres formas (forma de L, paralelogramo y hexágono) y tres colores (azul, naranja y marrón) adicionales. Se extrajeron seis parejas de figuras semejantes en color del conjunto correspondiente y se correlacionaron con seis arreglos de igualación de la muestra -extraídos al azar del conjunto recién mencionado-, y estos seis ensayos de igualación se usaron en los ensayos de preprueba y de prueba de generalización.

Además de esto, se utilizó un procedimiento similar para construir seis ensayos en los que la relación de igualación fue de semejanza en forma y seis ensayos en los que la relación de igualación fue de diferencia. Los ensayos restantes de la preprueba y la prueba de generalización se extrajeron al azar del primer y del segundo bloques de ensayos de entrenamiento: seis ensayos de semejanza en color y seis ensayos de semejanza en forma.

En resumen, la preprueba y la prueba de generalización fueron tareas idénticas, con 36 ensayos de igualación de la muestra; la mitad de ellos elaborados con las figuras y colores utilizados en el entrenamiento (estímulos familiares) y la otra mitad con figuras y colores que no se utilizaron en el entrenamiento (estímulos no familiares); en cada caso hubo seis ensayos de semejanza en color, seis de semejanza en forma y seis en los que la relación de igualación vigente fue la diferencia. La fase de entrenamiento consistió en dos bloques de 36 ensayos de igualación de la muestra de segundo orden, en los que la posición espacial de los cuatro tipos de estímulos de comparación (el idéntico, el semejante en color, el semejante en forma y el diferente en forma y color) cambió aleatoriamente entre los ensayos. Para garantizar las variaciones en las funciones discriminativa y delta de los estímulos, se utilizó entre los bloques de ensayos del entrenamiento una figura particular como estímulo de comparación correcto en un ensayo de igualación de semejanza en forma, y como incorrecto en un ensayo de semejanza en color.

\section{Procedimiento}

Los participantes de todos los grupos se expusieron a una preprueba, una fase de entrenamiento y dos tipos de pruebas de generalización cuyos ensayos se presentaron de forma aleatoria. No se impuso ningún criterio de ejecución para acceder a los ensayos de prueba de generalización con el fin de garantizar condiciones de entrenamiento homogéneas entre los grupos -excepto por las variables de retroalimentación-. Las respuestas de igualación correctas e incorrectas produjeron una pantalla en blanco de $5 \mathrm{~s}$ de duración para los participantes de todos los grupos a lo largo de los ensayos de preprueba, así como a lo largo de los ensayos de prueba de generalización. Las instrucciones que precedieron los ensayos de preprueba y de la prueba de generalización fueron:

"En las siguientes pantallas aparecerán siete figuras geométricas: dos en la parte superior, una en el centro y cuatro en la parte inferior. Debes elegir la figura de la parte inferior que creas que va con la figura del centro, de acuerdo con lo que indican las figuras de la parte superior. Para registrar tu elección, coloca el puntero del mouse dentro de la figura que elegiste y presiona el botón izquierdo. Para iniciar, haz clic en el botón COMENZAR".

Instrucciones similares precedieron a los ensayos de entrenamiento para la mayoría de los grupos. Durante el entrenamiento, las respuestas correctas e incorrectas 
produjeron la retroalimentación correspondiente en cada ensayo de igualación para los participantes del Grupo Retroalimentación Continua Correcto-Incorrecto, y cada tres ensayos para los participantes del Grupo Retroalimentación Intermitente Correcto-Incorrecto. La retroalimentación para las respuestas de igualación correctas e incorrectas consistió, respectivamente, en la presentación de las palabras "Correcto" e "Incorrecto" durante $5 \mathrm{~s}$ en el centro de la pantalla de la computadora. Para los participantes del Grupo Retroalimentación Correcto-Nada, las respuestas de igualación correctas produjeron la palabra "Correcto" en el centro de la pantalla durante $5 \mathrm{~s}$, mientras que las respuestas de igualación incorrectas produjeron una pantalla en blanco de $5 \mathrm{~s}$ de duración. Para los participantes del Grupo Retroalimentación Incorrecto-Nada, las respuestas de igualación correctas produjeron una pantalla en blanco de $5 \mathrm{~s}$, mientras que las respuestas incorrectas produjeron la palabra "Incorrecto" durante $5 \mathrm{~s}$. Los participantes del Grupo Retroalimentación Correcto-Nada con Instrucciones y los del Grupo Retroalimentación Incorrecto-Nada con Instrucciones se expusieron a combinaciones de retroalimentación similares a las utilizadas para los participantes de los grupos Retroalimentación Correcto-Nada y Retroalimentación Incorrecto-Nada, respectivamente; sin embargo, se les instruyó sobre el significado de las pantallas en blanco antes del inicio del entrenamiento:

"En las siguientes pantallas aparecerán siete figuras geométricas: dos en la parte superior, una en el centro y cuatro en la parte inferior. Debes elegir la figura de la parte inferior que creas que va con la figura del centro, de acuerdo con lo que indican las figuras de la parte superior. Te informaremos si tus elecciones fueron correctas o incorrectas. La palabra CORRECTO [INCORRECTO] seguirá a una elección correcta [incorrecta], mientras que una pantalla en blanco seguirá una elección incorrecta [correcta]. Para registrar tu elección, coloca el puntero del mouse dentro de la figura que elegiste y presiona el botón izquierdo. Para iniciar, haz clic en el botón COMENZAR".

\section{Resultados}

La Figura 1 muestra el porcentaje promedio de respuestas correctas en los ensayos de preprueba (barras blancas) y de prueba de generalización (barras negras), así como la ejecución a lo largo de la fase de entrenamiento en bloques de 18 ensayos para cada participante; las líneas discontinuas horizontales indican el $80 \%$ de respuestas correctas. En detalle, se muestra que, con excepción de un participante del Grupo Retroalimentación Incorrecto-Nada (P16), las ejecuciones oscilaron entre cero y $50 \%$ de respuestas correctas para la mayoría de los participantes en los ensayos de preprueba; que las ejecuciones promedio en los ensayos de prueba de generalización oscilaron entre 22 y $38 \%$ de respuestas correctas para los participantes del Grupo de Retroalimentación Continua Correcto-Incorrecto, mientras que para la mayoría de los participantes del Grupo Retroalimentación Intermitente Correcto-Incorrecto oscilaron entre 88 y $100 \%$ de respuestas correctas; y que la excepción fue P8, cuya ejecución en las pruebas de generalización fue del $22 \%$ de respuestas correctas.

Adicionalmente, las ejecuciones en la prueba de generalización estuvieron entre el 16 y el $66 \%$ de respuestas correctas para los participantes del Grupo Retroalimentación Correcto-Nada, mientras que para la mayoría de los participantes del Grupo Retroalimentación Incorrecto-Nada las ejecuciones oscilaron entre 88 y $100 \%$ de respuestas correctas. En este caso, la excepción fue P8, cuya ejecución en las pruebas de generalización fue del $66 \%$ de respuestas correctas. Por otra parte, las ejecuciones terminales oscilaron entre cero y $50 \%$ de respuestas correctas para la mayoría de los participantes de los dos grupos restantes, y la excepción fue P23, cuya ejecución en los ensayos de generalización fue del $63 \%$ de respuestas correctas. Con respecto a la fase de entrenamiento, la Figura 1 muestra que las ejecuciones estuvieron alrededor o por debajo del $50 \%$ de respuestas correctas para la mayoría de los participantes y los bloques de ensayos.

Asimismo, excepto por algunos participantes de diferentes grupos (P13, P23 y 29), solo los participantes de los grupos Retroalimentación Intermitente Correcto-Incorrecto (excepto P8) y Retroalimentación Incorrecto-Nada mostraron ejecuciones superiores al $50 \%$ de respuestas correctas en dos o más bloques de ensayos de entrenamiento.

Por otra parte, la Figura 2 muestra los porcentajes individuales de respuestas correctas por relación de igualación en los ensayos de prueba de generalización con estímulos familiares (barras negras) y no familiares (barras blancas). La inspección visual de la Figura 2 sugiere: (a) que porcentajes altos de respuestas correctas en los ensayos de prueba de generalización en los que la relación de igualación vigente fue de diferencia se observaron solamente para los participantes de los grupos Retroalimentación Intermitente Correcto-Incorrecto y Retroalimentación Incorrecto-Nada (excepto P30 con estímulos familiares); (b) que las ejecuciones fueron relativamente independientes del carácter familiar o no familiar de los estímulos utilizados; y (c) que la semejanza en forma parece ser una relación de igualación ligeramente menos difícil que la semejanza en color. 


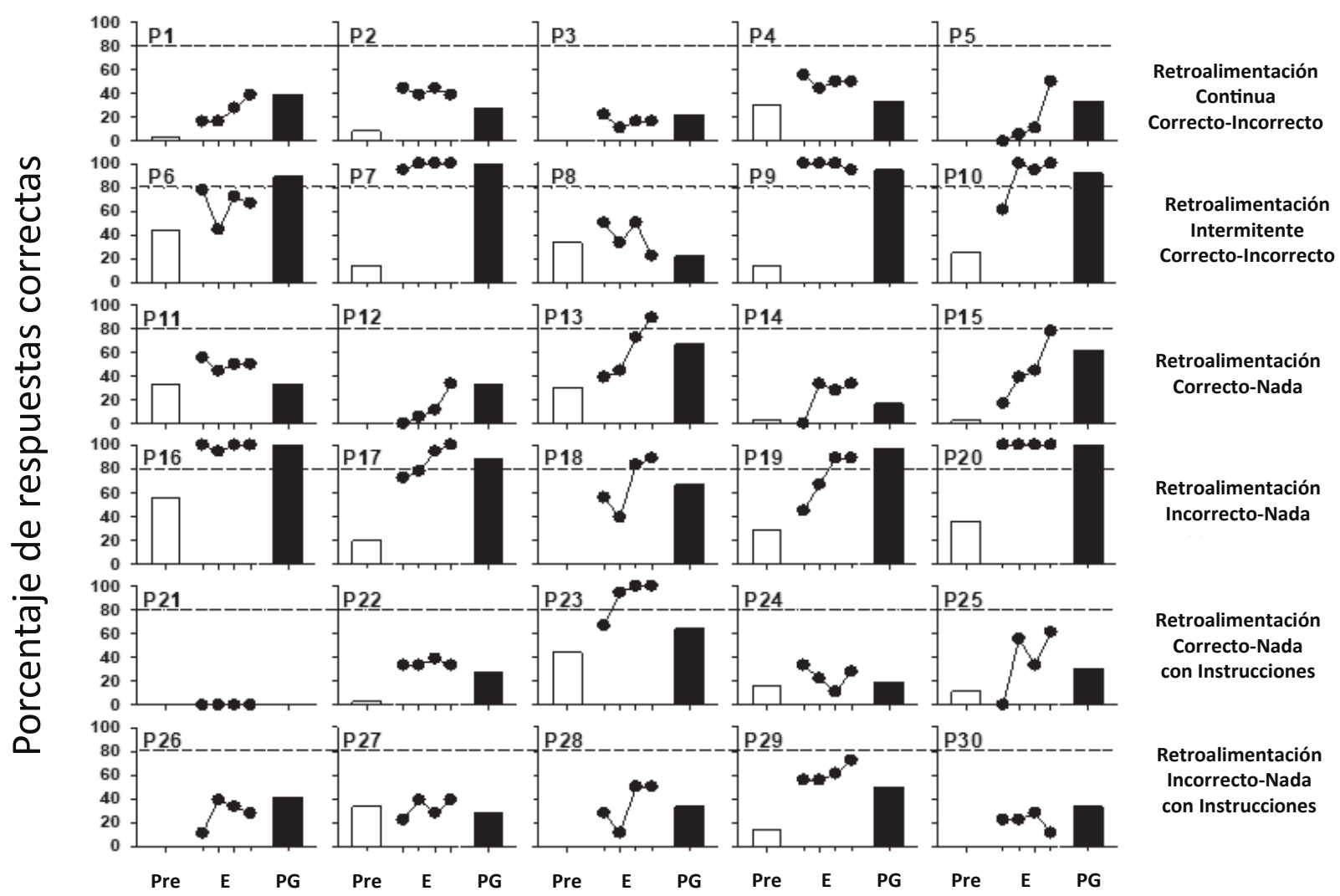

Figura 1. Porcentaje de respuestas correctas en la preprueba (barras blancas), el entrenamiento [bloques de 18 ensayos (puntos)] y prueba de generalización (barras negras) para cada participante. Nota: Pre = preprueba; E = Entrenamiento; $\mathrm{PG}=$ Ensayos de prueba de generalización; línea punteada $=80 \%$.

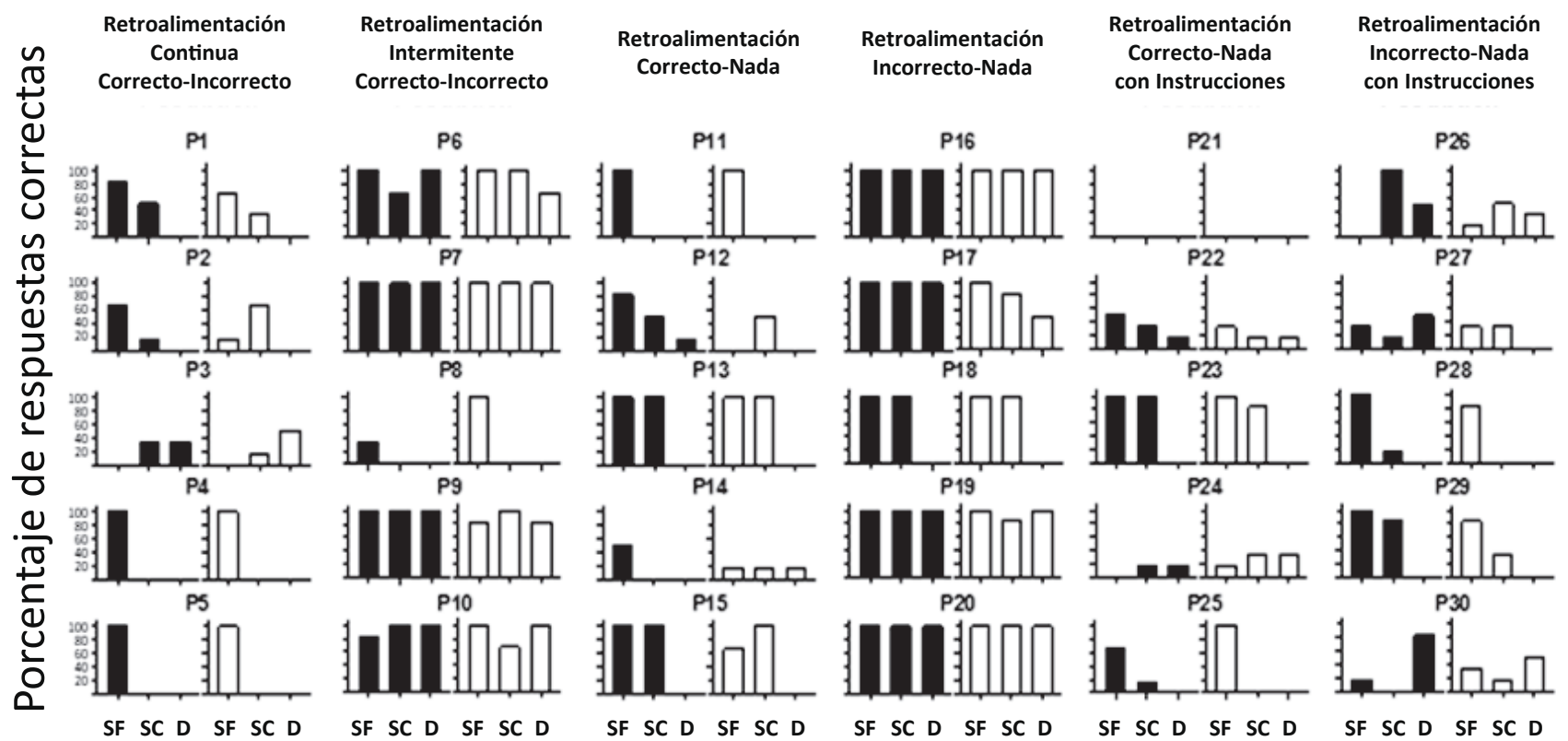

Figura 2. Porcentaje de respuestas correctas por relación de igualación en los ensayos de prueba de generalización con estímulos familiares (barras negras) y no familiares (barras blancas) para cada participante. Nota: $\mathrm{SF}=$ semejanza en forma; $\mathrm{SC}=$ semejanza en color; $\mathrm{D}=$ diferencia. 


\section{Discusión}

La retroalimentación intermitente para las respuestas de igualación correctas e incorrectas (grupo Retroalimentación Intermitente Correcto-Incorrecto) durante el entrenamiento de una tarea de igualación de la muestra de segundo orden produjo una mayor precisión del responder en pruebas de generalización que la retroalimentación continua (grupo Retroalimentación Continua Correcto-Incorrecto); mientras que la retroalimentación y las pantallas en blanco para las respuestas de igualación incorrectas y correctas, respectivamente (grupo Retroalimentación Incorrecto-Nada), produjeron una mayor ejecución de igualación de la muestra generalizada "intra-relacional" (como en los ensayos de semejanza en forma y semejanza en color) en comparación con la combinación inversa de retroalimentación y pantallas en blanco (como en el grupo Retroalimentación Correcto-Nada), además de una ejecución generalizada "extra-relacional" similar a la observada bajo la condición de retroalimentación intermitente Correcto-Incorrecto.

Adicionalmente, y como se esperaba, las instrucciones sobre el significado de las pantallas en blanco (ambos grupos con instrucciones) produjeron ejecuciones similares a las observadas en la condición en la que las respuestas de igualación correctas e incorrectas produjeron la retroalimentación correspondiente; sin embargo, la precisión de las respuestas bajo estas tres condiciones fue tan baja como la observada bajo la condición en la que las respuestas de igualación correctas e incorrectas produjeron la retroalimentación correspondiente y con pantallas en blanco, respectivamente.

Los resultados inmediatamente anteriores sugieren que, aunque efectivamente "puede ser engañoso hablar de las propiedades reforzantes de estar en lo correcto y las propiedades de castigo de estar en lo incorrecto" (Catania, 2006, p. 298), proporcionar información sobre el carácter preciso (o impreciso) del comportamiento de un individuo no es necesariamente equivalente a una instrucción como "responde (o no respondas) de la misma manera la próxima vez". De hecho, y parcialmente en contraste con la hipótesis expresada por Spence (1964) con respecto al significado de las pantallas en blanco, los resultados del presente experimento sugieren que las instrucciones sobre la potencial función de los eventos pueden obstruir su actualización, probablemente debido a una "confusión" similar a la que según dicha autora parece tener lugar bajo la combinación de retroalimentación Correcto-Nada, o bien debido al tipo de contingencias implicadas en la tarea experimental y sus características particulares. Nuevos experimentos deberían evaluar ambas posibilidades.
Por otra parte, el hecho de que la retroalimentación intermitente produjera mayor ejecución de igualación de la muestra generalizada que la retroalimentación continua es consistente con la idea de que esta última favorece el control por parte de las propiedades particulares de los estímulos de muestra y de comparación, mientras que la ausencia de retroalimentación en algunos ensayos de igualación promueve el desligamiento de las contingencias momento a momento (Martínez \& Ribes, 1996).

De acuerdo con la propuesta taxonómica de Ribes y López (1985), dado un lenguaje disponible, dicho desligamiento tiene lugar como una interacción lingüística con respecto al propio desempeño y a las contingencias enfrentadas (" $i \mathrm{El}$ concepto es la semejanza!", por ejemplo) y -debido a sus propiedades funcionales dentro de un medio de contacto convencional- permite, además, la introducción de otro estímulo discriminativo verbal pertinente para una situación no entrenada de solución de problemas en ese dominio particular (“¡La nueva relación es diferencia!”, por ejemplo).

Asimismo, dado que los participantes del Grupo Retroalimentación Incorrecto-Nada mostraron ejecuciones de igualación de la muestra generalizada similares a los observados para los participantes del Grupo Retroalimentación Intermitente Correcto-Incorrecto bajo ensayos de prueba en los que la relación de igualación vigente fue la de diferencia, los resultados del presente experimento sugieren que tuvo lugar un proceso de desligamiento funcional similar al descrito previamente para la retroalimentación intermitente Correcto-Incorrecto, cuando las respuestas incorrectas y correctas produjeron retroalimentación y pantallas en blanco, respectivamente (Serrano et al., 2017).

De igual forma, sobre la base de las ejecuciones observadas para los participantes de los grupos Retroalimentación Incorrecto-Nada y Retroalimentación Correcto-Nada, los resultados del presente experimento también coinciden con la hipótesis expresada por Spence (1964) sobre una tendencia inicial de los participantes de tratar las pantallas en blanco como si significaran Correcto bajo ambos tipos de combinaciones de retroalimentación y pantallas en blanco y, en correspondencia, la incompatibilidad de la combinación de retroalimentación Correcto-Nada con respecto a las demandas de la tarea.

El hecho de que en el presente experimento los participantes expuestos a retroalimentación continua mostraran ejecuciones severamente bajas probablemente pudo deberse al tipo de relaciones de igualación entrenadas. En este sentido, Serrano et al. (2009) informaron ejecuciones similares entre los participantes expuestos a retroalimentación continua y los participantes expuestos a retroalimentación y pantallas en blanco para las respuestas de igualación incorrectas y correctas, respectivamente; sin embargo, en 
60

sus experimentos las relaciones de igualación entrenadas incluyeron identidad y diferencia, relaciones que parecen ser menos difíciles de aprender que la relación de igualación de semejanza en color (véase también Ribes \& Torres, 2001). La diferencia entre los experimentos con respecto al número de estímulos de comparación desplegados en cada ensayo de igualación (tres versus cuatro) también pudo afectar la adquisición de la discriminación condicional por parte de los participantes del Grupo Retroalimentación Continua Correcto-Incorrecto del presente reporte. Nuevos experimentos deberían abordar ambas posibilidades.

En cualquier caso, debe destacarse que la precisión de la ejecución en los ensayos de prueba de generalización para los participantes expuestos a la retroalimentación continua fue tan baja como la precisión de respuesta observada para los participantes que recibieron instrucciones sobre el significado de las pantallas en blanco y expuestos a combinaciones de retroalimentación y pantallas en blanco. Estos resultados son importantes, por un lado, porque sugieren que las instrucciones iniciales sobre el significado de las pantallas en blanco efectivamente tornaron las combinaciones de retroalimentación Correcto-Nada e Incorrecto-Nada similares a la condición de retroalimentación continua Correcto-Incorrecto a lo largo de los ensayos de entrenamiento; $y$, por otro, porque -inesperadamente- ninguna de dichas condiciones de retroalimentación continua produjo un porcentaje relativamente alto de respuestas correctas en la fase de entrenamiento. Nótese, sin embargo, que se observó una ejecución generalizada extra-relacional incipiente para dos participantes del Grupo Retroalimentación Correcto-Nada con Instrucciones y tres participantes del Grupo Retroalimentación Incorrecto-Nada con Instrucciones, mientras que una ejecución generalizada extra-relacional incipiente no se observó para cuatro de los cinco participantes del Grupo Retroalimentación Continua Correcto-Incorrecto.

Por último, retomando el experimento realizado por Hirst et al. (2013) sobre la proporción de ensayos con retroalimentación precisa, los nuevos experimentos de retroalimentación continua versus intermitente e igualación de la muestra generalizada deberán evaluar los efectos de la probabilidad de retroalimentación a lo largo de los ensayos de entrenamiento; especialmente en tareas de igualación de la muestra sin estímulos de segundo orden (GonzálezBecerra \& Ortiz, 2014), así como a partir de diseños en los que sujetos ingenuos observen el desempeño de participantes expertos (Rodríguez, Silva, Baustista \& Peña, 2015) e interactúen con ellos lingüísticamente, como un modelo experimental de la enseñanza escolar.

\section{Referencias}

Catania, A. C. (2006). Learning (Interim [4th] Ed.). Cornwallon-Hudson, NY: Sloan Publishing.

González-Becerra, V., \& Ortiz, G. (2014). Efectos del tipo de contenido de las descripciones pre-contacto sobre la conducta de discriminación condicional y las descripciones post-contacto. Acta Colombiana de Psicología, 17(1), 1123. doi: 10.14718/ACP.2014. 17.1.2

Hirst J. M., DiGennaro-Reed, F. D., \& Reed, D. D. (2013). Effects of varying feedback accuracy on task acquisition: A computerized translational study. Journal of Behavioral Education, 22, 1-15. doi: 10.1007/s10864-012-9162-0

Mangiapanello, K. A., \& Hemmes, N. S. (2015). An analysis of feedback from a behavior analytic perspective. The $B e$ havior Analyst, 38, 51-75. doi: 10.1007/s40614-014-0026-x

Martínez, H., \& Ribes, E. (1996). Interactions of contingencies and instructional history on conditional discrimination. The Psychological Record, 46, 301-318. Recuperado de http:// psycnet.apa.org/record/1996-00325-005

Ribes, E., \& López, F. (1985). Teoría de la conducta: Un análisis de campo y paramétrico. México: Trillas.

Ribes, E., \& Torres, C. (2001). Un estudio comparativo de los entrenamientos de primer y segundo orden en igualación de la muestra. Revista Mexicana de Análisis de la Conducta, 27, 385-401.

Rodríguez, M. E., Silva, L. H., Bautista, L. R., \& Peña, T. E. (2015). Efectos de diferentes tipos de entrenamiento en el aprendizaje de una discriminación condicional, Acta Colombiana de Psicología 18(1), 55-67. doi: 10.14718/ ACP.2015. 18.1.6

Serrano, M., Flores, C., Peralta, S., \& Martínez, D. (2017). Efectos de la retroalimentación para las respuestas correctas o incorrectas en igualación de la muestra de segundo orden. Revista Argentina de Ciencias del Comportamiento, 9, 1-14. doi:10.30882/1852.4206.v9.n3.17242

Serrano, M., García, G., \& López, A. (2009). Efectos de la retroalimentación para las respuestas de igualación correctas o incorrectas en la adquisición y transferencia de discriminaciones condicionales. Revista Mexicana de Análisis de la Conducta, 35, 113-134.

Skinner, B. F. (1950). Are theories of learning necessary? Psychological Review, 57, 193-216. doi: 10.1037/h0054367

Spence, J. T. (1964). Verbal discrimination performance under different verbal reinforcement combination. Journal of Experimental Psychology, 67, 195-197. doi: 10.1037/ h0045522 


\title{
Combinaciones de retroalimentación e igualación de la muestra generalizada bajo estímulos y relaciones de igualación familiares y no familiares
}

\author{
MARIO SERRANO* \\ Universidad Veracruzana, CEICAH, Veracruz, México \\ ORCID: https://orcid.org/0000-0002-1190-3024 \\ CARLOS FLORES \\ Universidad de Guadalajara, CEIC, Jalisco, México \\ ORCID: https://orcid.org/0000-0002-3085-4029
}

\begin{abstract}
How to quote this article: Serrano, M. \& Flores, C. (2019). Feedback combinations and generalized matching-to-sample performance under familiar and unfamiliar stimuli and matching relations. Acta Colombiana de Psicología, 22(1), 61-68. doi: http://www.dx.doi.org/10.14718/ACP.2019.22.1.4
\end{abstract}

Resumen

\begin{abstract}
Seis grupos de estudiantes de preparatoria se expusieron a una tarea de igualación de la muestra de segundo orden y a pruebas de generalización con estímulos familiares y no familiares, así como con una nueva relación de igualación. Para dos grupos, las respuestas de igualación correctas e incorrectas produjeron la retroalimentación correspondiente de acuerdo con un programa continuo y uno intermitente, respectivamente. Las respuestas correctas produjeron retroalimentación y las respuestas incorrectas produjeron pantallas en blanco y viceversa para otros dos grupos, respectivamente. Dos grupos adicionales estuvieron expuestos a combinaciones similares de retroalimentación y pantallas en blanco, pero se instruyó a los participantes sobre el "significado" de las pantallas antes del entrenamiento. Se observó igualación de la muestra generalizada extra-relacional con estímulos familiares o no familiares solo después de las condiciones de entrenamiento en las que se programó retroalimentación intermitente Correcto-Incorrecto, así como cuando las respuestas de igualación incorrectas produjeron pantallas en blanco y las respuestas correctas produjeron la retroalimentación correspondiente. Las instrucciones sobre el significado de las pantallas en blanco produjeron ejecuciones generalizadas ligeramente superiores a las observadas después de la retroalimentación continua Correcto-Incorrecto, las cuales a su vez fueron similares a las ejecuciones observadas después de la condición Correcto-Pantalla en blanco, sin instrucción. Los resultados confirman una tendencia inicial a tratar las pantallas en blanco como retroalimentación para respuestas correctas y sugieren un proceso común de "desligamiento" entre la retroalimentación intermitente y la retroalimentación Incorrecto-Pantalla en blanco.

Palabras clave: control abstracto del estímulo, discriminación condicional, igualación de la muestra generalizada, humanos.
\end{abstract}

\section{Feedback combinations and generalized matching-to-sample performance under familiar and unfamiliar stimuli and matching relations}

\begin{abstract}
Six groups of high-school students were exposed to a second-order matching-to-sample task and generalization tests trials using familiar and unfamiliar stimuli as well as a new matching relation. For two groups correct and incorrect matching responses produced the corresponding feedback according to continuous and intermittent schedules, respectively. Correct responses produced feedback and incorrect responses produced blanks and vice versa for other two groups, respectively. Two additional groups were exposed to similar feedback-blanks combinations but participants were instructed about the "meaning" of blanks before training. Extra-relational generalized matching-to-sample performance with either familiar or unfamiliar stimuli was observed after training conditions in which intermittent right-wrong feedback was scheduled, as well as when incorrect matching responses produced blanks and correct responses produced the corresponding feedback.
\end{abstract}

\footnotetext{
Universidad Veracruzana, Centro de Estudios e Investigaciones en Conocimiento y Aprendizaje Humano. Av. Orizaba N. ${ }^{\circ}$ 203, Fraccionamiento Veracruz, Xalapa, Veracruz, México. C. P. 91020. Teléfono: +52 (228) 89034 65. mserrano@uv.mx

Partes de este trabajo se presentaron en la "42nd Annual Convention of the Association for Behavior Analysis International". La investigación se condujo durante el año sabático del primer autor en la Universidad de Guadalajara, bajo la supervisión del segundo autor. Se agradece al Dr. Oscar García Leal por facilitar la infraestructura para las actividades académicas y de investigación.
} 
Instructions about the meaning of blanks produced generalized performances slightly higher to those observed after continuous right-wrong feedback, which in turn were similar to performances observed after the uninstructed right-blank feedback combination condition. Results confirm an initial tendency to treat blanks as if they mean right and suggest a common "detachment" processes between intermittent feedback and the wrong-blanks feedback combination.

Keywords: abstract stimulus control, conditional discrimination, generalized matching-to-sample, humans.

\title{
Combinaçóes de retroalimentação e igualação da amostra generalizada sob estímulos e relaçóes de igualaçáo familiares e náo familiares
}

\author{
Resumo
}

\begin{abstract}
Seis grupos de estudantes de vestibular foram expostos a uma tarefa de igualação da amostra de segunda ordem e a testes de generalização com estímulos familiares e não familiares, bem como a uma nova relação de igualação. Para dois grupos, as respostas de igualação corretas e incorretas produziram a retroalimentação correspondente de acordo com um programa contínuo e um intermitente, respectivamente, em que as respostas corretas produziram retroalimentação e as incorretas, ecrãs brancos - e vice-versa para outros dois grupos. Dois grupos adicionais estiveram expostos a combinações semelhantes de retroalimentação e ecrãs brancos, mas foi instruído aos participantes o "significado" das telas antes do treinamento. Foi observada igualação da amostra generalizada extrarrelacional com estímulos familiares e não familiares somente depois das condições de treinamento nas quais foi programada a retroalimentação intermitente Correto-incorreto, bem como quando as respostas de igualação incorretas produziram ecrãs brancos, e quando as respostas corretas produziram a retroalimentação correspondente. As instruções sobre o significado dos ecrãs brancos produziram execuções generalizadas levemente superiores às observadas após a retroalimentação contínua Correto-incorreto, as quais, por sua vez, foram similares às execuções observadas depois da condição Correto-ecrã branco, sem instrução. Os resultados confirmam uma tendência inicial a tratar os ecrãs brancos como retroalimentação para respostas corretas e sugerem um processo comum de "desligamento" entre a retroalimentação intermitente e a retroalimentação Incorreto-ecrã branco.

Palavras-chave: controle abstrato do estímulo, discriminação condicional, igualação da amostra generalizada, humanos.
\end{abstract}

\section{Introduction}

In a conditional discrimination situation, discriminative and delta functions of stimuli varies from one moment to the other conditionally upon a third, previous or concurrent stimulus segment. In terms of the typical matching-to-sample procedure with pigeons originally described by Skinner (1950), the third stimulus segment is the sample stimulus while comparison stimuli are the stimulus objects whose functions varies from trial to trial. Different matching relations could be trained on the basis of the physical properties of stimuli (e.g., identity, difference, similarity, etc.). In the case of human beings, after learning to perform according to one or more matching relations, participants may immediately match unfamiliar sample and comparison stimuli according with such criteria. If so, the performance is said to be generalized because it is not restricted to the specific trained stimuli or their physical properties.

As in natural settings, responding under matching-tosample procedures could be influenced by feedback, that is, exteroceptive stimuli regarding the correspondence between an individual's behavior and prior or ongoing reinforcement contingencies (Mangiapanello \& Hemmes, 2015). In a recent experiment, for example, Hirst, DiGennaro Reed and Reed (2013) explored the effects of feedback accuracy upon the acquisition of an arbitrary conditional discrimination by young adults. In their study, groups of participants received feedback that their matching responses were correct following incorrect choices and vice versa on $25,50,75$ and $100 \%$ of the trials. They observed that the higher the proportion of inaccurate feedback trials, the lower the acquisition of the conditional discrimination in a second phase with accurate feedback; suggesting that feedback accuracy establishes the discriminative relation between a stimulus situation and behavior in a similar way to instructions or contingency-specifying stimuli. In this sense, Catania (2006) previously pointed out that providing information to an individual about that his response was correct is likely to say "respond the same way next time", suggesting that "it may be misleading to speak of the reinforcing properties of being correct and the punishing properties of being incorrect" (p. 298).

Using a verbal learning discrimination task, Spence (1964) reported that performance along training trials was 
lower under a Right-Nothing feedback combination than under a Wrong-Nothing feedback combination, which in turn produced similar performances to those observed under the typical Right-Wrong feedback condition. In agreement with the idea expressed by Catania (2006), she suggested that the dissimilar effects of Right-Nothing and Wrong-Nothing feedback combinations were due to the "informative" characteristics of nothing (i.e., blanks) rather than to a difference in positive and negative reinforcement functions of right and wrong, respectively. More specifically, Spence proposed that in the absence of instructions about its meaning, participants tend to treat blanks as if it meant right under both kind of feedback combinations. Being that way, such tendency is incompatible with the demands of the experimental task to treat blanks as incorrect under the Right-Nothing feedback combination, while under the Wrong-Nothing feedback combination the tendency promotes conditions similar to those in which correct and incorrect responses produce the correspondently feedback in each trial.

Previous experiments on generalized matching-tosample, however, suggest that the absence of feedback in some trials has a more complex effect upon behavior. For example, Martínez and Ribes (1996) reported that participants exposed to an intermittent (i.e., every third trial) Right-Wrong feedback condition were less controlled by initial false instructions regarding the correct matching relation in both training and generalization tests than participants exposed to continuous Right-Wrong feedback. A third condition of delayed feedback (i.e., at the end of each training session) produced an intermediate performance. Martínez and Ribes suggested that the absence of feedback in some matching trials prompted a "detachment" from moment-to-moment contingencies and, accordingly, the self-construction of an abstract verbal discriminative stimulus controlled by the functional propriety shared by stimuli along trials (i.e., the matching relation).

Using matching-to-sample tasks with both Right-Nothing and Wrong-Nothing feedback combinations as well as the typical continuous Right-Wrong feedback condition, Serrano, García, and López (2009) reported that generalized matching-to-sample performance agree with the hypothesis expressed by Spence (1964) that participants tend to treat nothing as if it meant right along training trials. More recently, Serrano, Flores, Peralta and Martínez (2017) observed a higher generalized matching-to-sample performance after a Wrong-Nothing feedback combination training condition than after a Right-Nothing one, but also that the Wrong-Nothing feedback combination produced a higher generalized accuracy than continuous Right-Wrong feedback when generalization tests contained an untrained matching relation. By virtue of the performance observed under such "extra-relational" generalization tests trials, these latter authors suggested that their results were due to the initial tendency to treat nothing as if it meant right, but also to a detachment from moment-to-moment contingencies similar to that described by Martínez and Ribes (1996) regarding intermittent Right-Wrong feedback. The experiment, however, did not include a condition in which correct and incorrect responses produced feedback in an intermittently fashion.

On the one hand, the present experiment was conducted in order to compare the effects of a Wrong-Nothing feedback combination versus intermittent Right-Wrong feedback upon generalized matching-to-sample performance. If the effects of the Wrong-Nothing feedback combination are the result of an initial tendency to treat nothing as if it meant right but also the promotion of self-constructed verbal stimulus due to a detachment from moment-to-moment contingencies, participants trained under a Wrong-Nothing feedback combination should show similar generalized performances to those observed for participants exposed to intermittent Right-Wrong feedback, which in turn should be higher than performances observed for participants trained under continuous Right-Wrong feedback. On the other hand, it must be noted that in contrast with the hypothesis expressed by Spence (1964), neither available experiment on feedback combinations and generalized matchingto-sample had included participants instructed about the meaning of blanks before training. Following the suggestion expressed by Catania (2006), if right and wrong have an instructional function like "respond (or do not respond) the same way next time", participants instructed about the meaning of blanks and trained under Right-Nothing and Wrong-Nothing feedback combinations should show generalized performances similar to those observed for participants exposed to continuous Right-Wrong feedback.

\section{Method}

\section{Participants}

Participants were 30 high-school students aged 17 to 18 years, 21 female and 9 male, experimentally naïve in conditional discrimination tasks as well as in any other experimental procedure in Psychology. All participants provided written informed consent. Five participants were randomly assigned to each of six groups: Continuous Right-Wrong Feedback, Intermittent Right-Wrong Feedback, RightNothing Feedback, Wrong-Nothing Feedback, Instructed 
Right-Nothing Feedback, and Instructed Wrong-Nothing Feedback.

\section{Apparatus and experimental settings}

Participants worked individually in different small (3$\mathrm{m} 2$ ), quiet rooms. Each of the five rooms was equipped with a table, a chair, a desktop computer (HP Compaq Model, dc5850) and a mouse. Stimuli were presented via the computer's monitor and the mouse served as the response device. Experimental events were automatically controlled and recorded using Superlab® ${ }^{\circledR} .5$.

Simultaneous second-order matching-to-sample trials were used for all groups of participants along the experiment. Each second-order matching-to-sample trial displayed seven colored shapes. Two colored shapes were the second-order stimuli that visually modeled the ongoing matching relation in each trail, one colored shape was the sample stimulus and the remaining four colored shapes were comparison stimuli. Second-order stimuli were displayed at the top of the computer's monitor, while sample and comparison stimuli were displayed at the center and bottom, respectively. Second-order stimuli were always different in shape and color from sample and comparison stimuli. In each matching trail one comparison stimulus was identical to the sample stimulus, one was similar in shape, another was similar in color and the fourth one was different in both color and shape. The spatial position of each kind of comparison stimulus was randomly changed from trial to trial along the experiment.

There were 36 pretest trials and 36 generalization test trials (12 color-similarity trials, 12 shape-similarity trials, and 12 difference trials), while there were 72 training trials (36 color-similarity trials and 36-shape similarity trials). Table 1 shows shapes and colors used to create second-order stimuli and first-order matching-to-sample arrangements (i.e., one sample and four comparison stimuli) used in each phase of the experiment.

Three shapes (rhombus, cross, and circle) and three colors (lightsky blue, gold, and black) allowed the construction of 18 pairs of stimuli similar in shape, 18 pairs of stimuli similar in color, and 36 pairs of stimuli different in both shape and color. Another set of three shapes (triangle, square, and pentagon) and three colors (green, yellow, and red) allowed the construction of 36 first-order matching-to-sample arrangements that were presented twice between two blocks of training trials. All pairs of colored shapes from color-similarity and shape-similarity pools of second-order stimuli were randomly correlated with first-order matching-to-sample arrangements in the
Table 1.

Shapes and colors used to create second-order stimuli and matching-to-sample arrangements in each phase of the experiment.

\begin{tabular}{|c|c|c|c|c|}
\hline \multirow[t]{2}{*}{ Stimuli } & \multicolumn{2}{|c|}{$\begin{array}{l}\text { Pretest and Generaliza- } \\
\text { tion trials }\end{array}$} & \multicolumn{2}{|l|}{ Training } \\
\hline & Shape & Color & Shape & Color \\
\hline \multirow{6}{*}{$\begin{array}{l}\text { Second- } \\
\text { order } \\
\text { stimuli }\end{array}$} & rhombus & $\begin{array}{l}\text { lightsky } \\
\text { blue }\end{array}$ & rhombus & $\begin{array}{l}\text { lightsky } \\
\text { blue }\end{array}$ \\
\hline & cross & gold & cross & gold \\
\hline & circle & black & circle & black \\
\hline & equal to & gray & & \\
\hline & chevron & purple & & \\
\hline & trapezoid & pink & & \\
\hline \multirow{6}{*}{$\begin{array}{l}\text { Sample and } \\
\text { comparison } \\
\text { stimuli }\end{array}$} & triangle & green & triangle & green \\
\hline & square & yellow & square & yellow \\
\hline & pentagon & red & pentagon & red \\
\hline & L shape & blue & & \\
\hline & parallelogram & orange & & \\
\hline & hexagon & brown & & \\
\hline
\end{tabular}

first block of training trials. Correlations between secondorder stimuli and matching-to-sample arrangements were inverted between training trials in the second block. Six pairs of stimuli different in both shape and color from the third pool of second-order stimuli were randomly extracted and correlated with six, randomly extracted matching-tosample arrangements. These six matching trials were used as pretest and generalization test trials.

A third set of three shapes (equal to, chevron, and trapezoid) and three colors (gray, purple, and pink) allowed the construction of three new pools of second-order stimuli similar to those just described. A second set of 36 matchingto-sample arrangements was constructed using additional three shapes (L-shape, parallelogram, and hexagon) and three colors (blue, orange, and brown). Six pairs of colored shapes similar in color were extracted from the corresponding pool and were correlated with six matching-to-sample arrangements, randomly extracted from the just mentioned set. These six matching trials were used as pretest and generalization test trials. A similar procedure was used to construct six trials in which the ongoing matching relation was shape-similarity and six trials in which the ongoing matching relation was difference. Remaining pretest and generalization test trials were six color-similarity and six shape-similarity arrangements randomly extracted from the first and the second blocks of training trials. 
Summarizing, pretest and generalization tests trials were identical tasks with 36 matching-to-sample trails; half constructed using the shapes and colors used in training (familiar stimuli) and the other half constructed with shapes and colors not used in training (unfamiliar stimuli). For each half there were six color-similarity trials, six shape-similarity trials, and six trials in which the ongoing matching relation was difference. The training phase consisted of two blocks of 36 second-order matching-to-sample trials in which the spatial position of the four kinds of comparison stimulus (i.e., the identical one, the one similar in color, the one similar in shape, and the one different in both color and shape) was randomly changed across trials. In order to ensure variations in discriminative and delta functions of stimuli, a particular colored shape was the correct comparison stimulus under a shape-similarity trial but an incorrect one under a colorsimilarity trial between blocks of training trials.

\section{Procedure}

Participants from all groups were exposed to pretest trials, a training phase, and two kinds of generalization tests trials that were presented in a mixed fashion across the test phase. No performance criterion was imposed to get access to generalization tests trials in order to ensure homogenous training conditions between groups (except for feedback variables). Correct and incorrect matching responses produced a $5 \mathrm{~s}$ long blank screen for participants from all groups along pretest trials as well as along generalization tests trials. Instructions before pretest and generalization test trials were:

Seven geometric figures will appear on the following screens: two at the top, one at the center and four at the bottom. You must choose the figure at the bottom that you think goes with the figure at the center, according to the indication by figures at the top. To record your choice, place the mouse pointer within the figure that you chose and press the left button. Click the START button to begin.

Similar instructions preceded training trials for most groups. During training, correct and incorrect responses produced the corresponding feedback every matching trial for participants from the Group Continuous Right-Wrong Feedback and every third trial for participants from the Group Intermittent Right-Wrong Feedback. Feedback for correct and incorrect matching responses respectively consisted of the presentation of the words "right" and "wrong" during 5 $\mathrm{s}$ on the center of the computer's monitor. For participants from the Group Right-Nothing Feedback, correct matching responses produced the word "right" in the center of the monitor during $5 \mathrm{~s}$, while incorrect matching responses produced a $5 \mathrm{~s}$ long blank screen. For participants from the
Group Wrong-Nothing Feedback, correct matching responses produced a $5 \mathrm{~s}$ long blank screen, while incorrect responses produced the word "wrong" during $5 \mathrm{~s}$. Participants from the Group Instructed Right-Nothing Feedback and those from the Group Instructed Wrong-Nothing Feedback were exposed to feedback combinations similar to those used for participant from groups Wrong-Nothing Feedback and Right-Nothing Feedback, respectively; however, they were instructed: as follows about the meaning of blanks before training began:

Seven geometric figures will appear on the following screens: two at the top, one at the center and four at the bottom. You must choose the figure at the bottom that you think goes with the figure at the center, according to the indication by the figures at the top. We will let you know if your choices were right or wrong. The word CORRECT [INCORRECT] will follow a right [wrong] choice, while a blank screen will follow a wrong [right] choice. To record your choice, place the mouse pointer within the figure that you chose and press the left button. Click the START button to begin.

\section{Results}

Figure 1 shows the mean percentage of correct responses on pretest (white bars) and generalization test (black bars) trails, as well as performance along the training phase in blocks of 18 trials for each participant. Dashed horizontal lines indicate $80 \%$ of correct responses. Figure 1 shows that except for one participant from the Group Wrong-Nothing Feedback (P16), performances were between zero and 50\% of correct responses for most participants in pretest trials. Mean performances in generalization test trials were between 22 and 38\% of correct responses for participants from the Group Continuous Right-Wrong Feedback, while for most participants from the Group Intermittent Right-Wrong Feedback performances were between 88 and $100 \%$ of correct responses. The exception was P8, whose performance in generalization tests trials was $22 \%$ of correct responses. Generalized performances were between 16 and $66 \%$ of correct responses for participants from the Group RightNothing Feedback, while for most participants from the Group Wrong-Nothing Feedback performances were between 88 and $100 \%$ of correct responses. In this case, the exception was $\mathrm{P} 8$, whose performance in generalization tests trials was $66 \%$ of correct responses. Terminal performances were between zero and $50 \%$ of correct responses for most participants from the remaining two groups. The exception was $\mathrm{P} 23$, whose performance in generalization tests trials was $63 \%$ of correct 


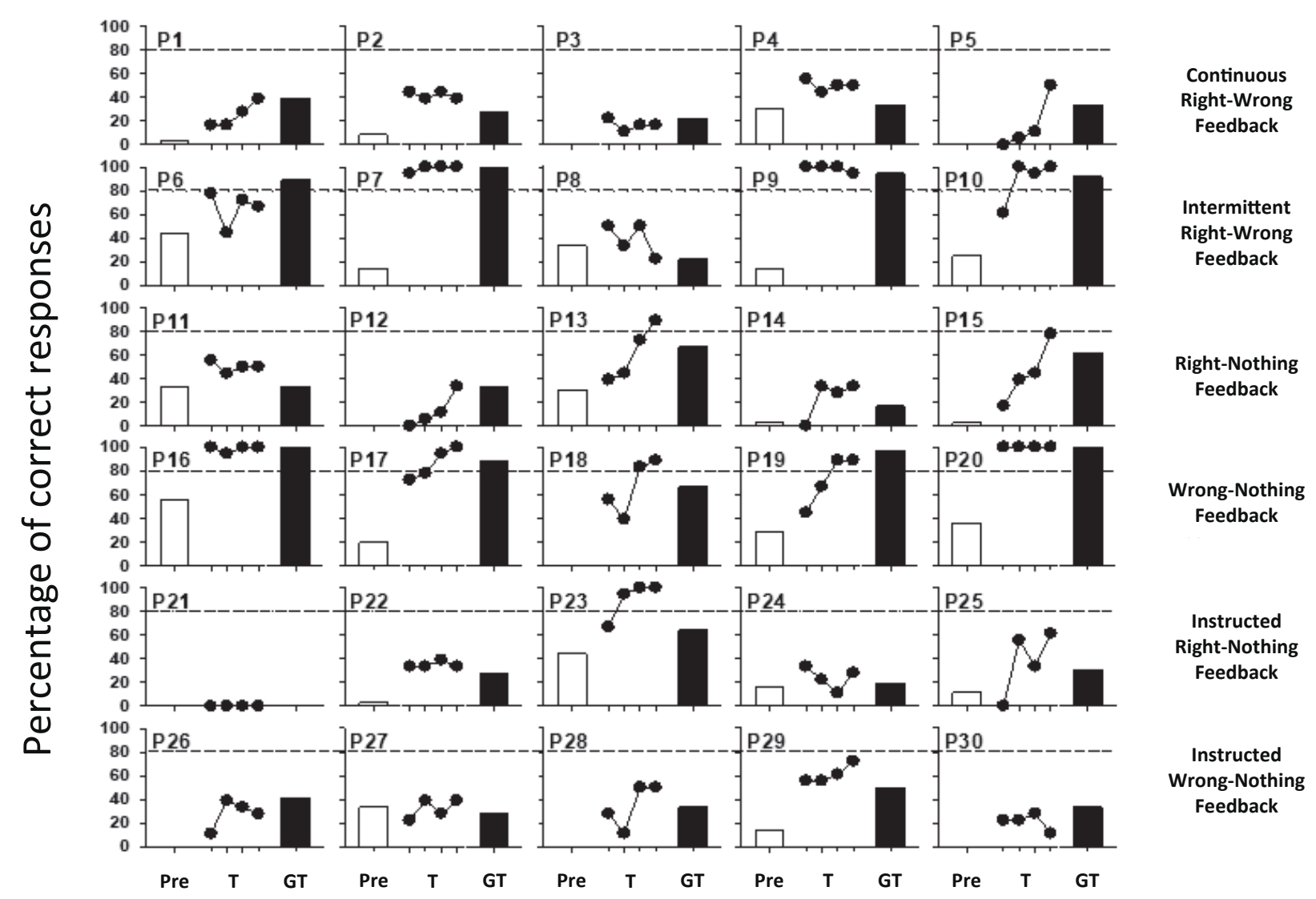

Figure 1. Percentage of correct responses in the pretest (white bars), training [blocks of 18 trials (dots)] and in generalization test (black bars) trails for each participant. Note: Pre $=$ pretest, $\mathrm{T}=$ training, $\mathrm{GT}=$ generalization test trials, and dashed lines $=80 \%$.

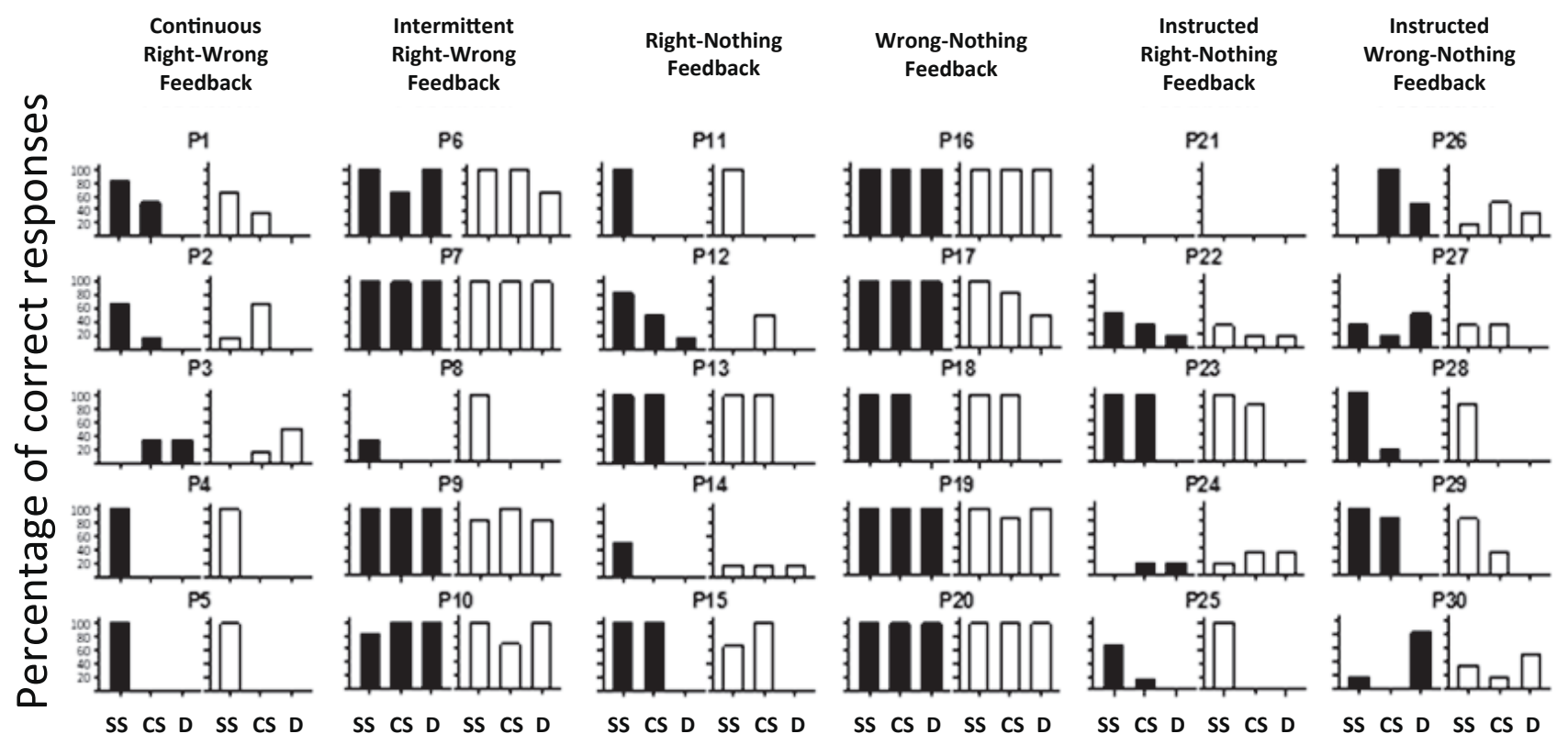

Figure 2. Percentage of correct responses per matching relation on generalization test trials with familiar (black bars) and unfamiliar (white bars) stimuli for each participant. Note: $\mathrm{SS}=$ shape-similarity, $\mathrm{CS}=$ color similarity, and $\mathrm{D}=$ difference. 
responses. Regarding the training phase, Figure 1 shows that performances were around or below $50 \%$ of correct responses for most blocks of trials and participants. In general, except for some participants from different groups (e.g., P13, P23, and 29), only participants from groups Intermittent RightWrong Feedback (except P8) and Wrong-Nothing Feedback showed performances above $50 \%$ of correct responses in two or more blocks of training trials.

Figure 2 shows individual percentages of correct responses per matching relation on generalization test trials with familiar (black bars) and unfamiliar (white bars) stimuli. Visual inspection of Figure 2 suggests that: a) high percentages of correct responses under generalization test trials in which the ongoing matching relation was difference were possible only for participants from groups Intermittent Right-Wrong Feedback and Wrong-Nothing Feedback (except for P30 with familiar stimuli); b) performances were relatively independent of the familiar or unfamiliar character of displayed stimuli; and c) shape-similarity seemed slightly less difficult matching relation than color-similarity.

\section{Discussion}

Intermittent feedback for both correct and incorrect matching responses (i.e., Group Intermittent Right-Wrong Feedback) during second-order matching-to-sample training produced a higher accuracy of responding in generalization tests trials than continuous feedback (i.e., Group Continuous Right-Wrong Feedback). Feedback and blanks for incorrect and correct matching responses (i.e., Group Wrong-Nothing Feedback), respectively, produced a higher "intra-relational" generalized matching-to-sample performance (i.e., under shape- and color-similarity trials) than the reverse feedbackblanks combination (i.e., Group Right-Nothing Feedback) and, moreover, an extra-relational generalized performance similar to that observed under the intermittent right-wrong feedback condition. As it was expected, instructions about the meaning of blanks (i.e., both instructed groups) produced performances similar to those observed under the condition in which both correct and incorrect matching responses produced the corresponding feedback; however, accuracy of responding under all these three conditions was as low as the accuracy observed under the condition in which correct and incorrect matching responses produced the corresponding feedback and blanks, respectively.

In particular, later results suggest that although effectively "it may be misleading to speak of the reinforcing properties of being correct and the punishing properties of being incorrect" (Catania, 2006, p. 298), providing information regarding the accurate (or inaccurate) character of an individual's behavior is not necessarily equivalent to an instruction like respond (or do not respond) the same way next time. In fact, and partially in contrasts with the hypothesis expressed by Spence (1964) regarding the meaning of blanks, results of the present experiment suggest that instructions about the potential function of events may obstruct its actualization; in this case, probably due to a similar "confusion" that according to such author seems to take place under the right-nothing feedback combination, or due to the kind of contingencies implied in the experimental task as well as its particular characteristics. New experiments should assess both possibilities.

The fact that intermittent feedback produced a higher generalized matching-to-sample performance in tests trials than continuous feedback is consistent with the idea that the later enhances control by particular properties of the sample and comparison stimuli, while the absence of feedback in some matching trials prompts a detachment from moment-to-moment contingencies (Martínez \& Ribes, 1996). According to the taxonomic proposal offered by Ribes and López (1985), being language available, such a detachment takes place as a linguistic interaction between participant's own performance and the ongoing contingencies (e.g., The concept is similarity!) and, due to their functional properties within a conventional medium of contact, additionally allows the introduction of other, pertinent verbal discriminative stimulus for an untrained problem-solving situation in that particular domain (e.g., The new relation is difference!). Given that participants from the Group Wrong-Nothing Feedback showed generalized matching-to-sample performances similar to those observed for participants from the Group Intermittent Right-Wrong Feedback under generalization test trials in which the ongoing matching relation was difference, results of the present experiment suggest that a functional detachment process similar to the one just described for intermittent right-wrong feedback had taken place when incorrect and correct responses produced feedback and blanks, respectively (Serrano et al. 2017). Based on performances observed for participants from groups Wrong-Nothing Feedback and Right-Nothing Feedback, results of the present experiment also agree with the hypothesis expressed by Spence (1964) about an initial tendency by participants to treat blanks as if they mean right under both kinds of feedback-blank combinations and, correspondingly, the incompatibility of the right-nothing feedback combination regarding the task's demands.

The fact that in the present experiment participants exposed to continuous feedback showed severely low performances probably was due to the kind of trained matching relations. Serrano et al. (2009) reported similar 
performances between participants exposed to continuous feedback and participants exposed to feedback and blanks for incorrect and correct responses, respectively. In their experiments, however, trained matching relations included identity and difference, which seemed to be less difficult to learn than the color- similarity matching relation (see also Ribes \& Torres, 2001). The difference between experiments regarding the number of displayed comparison stimuli per matching trial (three versus four) probably also affected the acquisition of the conditional discrimination by participants from the Group Continuous Right-Wrong Feedback in the present report. New experiments should address both possibilities.

In any case, it is noteworthy that accuracy of responding on generalization tests trials for participants exposed to continuous feedback was as low as accuracy of the performance observed for participants instructed about the meaning of blanks and exposed to feedback-blanks combinations. These results are important, on the one hand, because they suggest that the initial instructions about the meaning of blanks effectively turned right-nothing and wrong-nothing feedback combinations similar to the continuous rightwrong feedback condition along training trials and, on the other hand, because -unexpectedly- neither continuous feedback condition produced a relatively high percentage of correct responses in the training phase. Notice, however, that incipient extra-relational generalized performance was observed for two participants from the Group Instructed Right-Nothing Feedback and three participants from the Group Instructed Wrong-Nothing Feedback, while incipient extra-relational generalized responding was not observed for four of the five participants from the Group Continuous Right-Wrong Feedback.

Following the experiment conducted by Hirst et al. (2013) on the proportion of trials with accurate feedback, new experiments on continuous versus intermittent feedback and generalized matching to sample may assess the effects of feedback probability along training trials; especially under matching-to-sample tasks with no instructional secondorder stimuli (e.g., González-Berra \& Ortiz, 2014), as well as following designs in which naïve subjects observe the performance of expert participants (e.g., Rodríguez Pérez, Silva Castillo, Baustista Castro \& Peña Correal, 2015) and interact with them in an linguistically fashion, as an experimental model of scholar teaching.

\section{References}

Catania, A. C. (2006). Learning (Interim [4th] Ed.). Cornwallon-Hudson, NY: Sloan Publishing.

González-Becerra, V., \& Ortiz, G. (2014). Efectos del tipo de contenido de las descripciones pre-contacto sobre la conducta de discriminación condicional y las descripciones post-contacto. Acta Colombiana de Psicología, 17(1), 1123. DOI: 10.14718/ACP.2014. 17.1.2

Hirst J. M., DiGennaro Reed, F. D., \& Reed, D. D. (2013). Effects of varying feedback accuracy on task acquisition: A computerized translational study. Journal of Behavioral Education, 22, 1-15. DOI: 10.1007/s10864-012-9162-0

Mangiapanello, K. A., \& Hemmes, N. S. (2015). An analysis of feedback from a behavior analytic perspective. The Behavior Analyst, 38, 51-75. DOI: 10.1007/s40614-014-0026-x

Martínez, H., \& Ribes, E. (1996). Interactions of contingencies and instructional history on conditional discrimination. The Psychological Record, 46, 301-318.

Ribes, E., \& López, F. (1985). Teoría de la conducta: Un análisis de campo y paramétrico. México: Trillas.

Ribes, E., \& Torres, C. (2001). Un estudio comparativo de los entrenamientos de primer y segundo orden en igualación de la muestra. Revista Mexicana de Análisis de la Conducta, 27, 385-401.

Rodríguez Pérez, M. E., Silva Castillo, L. H., Bautista Castro, L. R., Peña Correal, T. E. (2015). Efectos de diferentes tipos de entrenamiento en el aprendizaje de una discriminación condicional, Acta Colombiana de Psicología 18(1), 55-67. DOI: 10.14718/ACP.2015. 18.1.6

Serrano, M., Flores, C., Peralta, S., \& Martínez, D. (2017). Efectos de la retroalimentación para las respuestas correctas o incorrectas en igualación de la muestra de segundo orden. Revista Argentina de Ciencias del Comportamiento, 9, 1-14.

Serrano, M., García, G., \& López, A. (2009). Efectos de la retroalimentación para las respuestas de igualación correctas o incorrectas en la adquisición y transferencia de discriminaciones condicionales. Revista Mexicana de Análisis de la Conducta, 35, 113-134.

Skinner, B. F. (1950). Are theories of learning necessary? Psychological Review, 57, 193-216. DOI: 10.1037/h0054367

Spence, J. T. (1964). Verbal discrimination performance under different verbal reinforcement combination. Journal of Experimental Psychology, 67, 195-197. DOI: 10.1037/ h0045522 